\title{
Development of an acrylamide-based inhibitor of protein S-acylation
}

Saara-Anne Azizi ${ }^{1,2, \#, ~ T o n g ~ L a n ~}{ }^{1, \#}$, Clémence Delalande ${ }^{1}$, Rahul S. Kathayat ${ }^{1}$, Bryan C. Dickinson ${ }^{1, *}$

${ }^{1}$ Department of Chemistry, The University of Chicago, Chicago, Illinois 60637, United States

${ }^{2}$ Medical Scientist Training Program, Pritzker School of Medicine, The University of Chicago, Chicago, Illinois 60637, United States

*Dickinson@uchicago.edu

\#These authors contributed equally to this work.

\begin{abstract}
Protein S-acylation is a dynamic lipid post-translational modification that can modulate the localization and activity of target proteins. In humans, the installation of the lipid onto target proteins is catalyzed by a family of 23 Asp-His-His-Cys domain-containing protein acyltransferases (DHHC-PATs). DHHCs are increasingly recognized as critical players in cellular signaling events and in human disease. However, progress elucidating the functions and mechanisms of DHHC "writers" has been hampered by a lack of chemical tools to perturb their activity in live cells. Herein, we report the synthesis and characterization of PATi, a panDHHC inhibitor more potent than 2-bromopalmitate (2BP), the most commonly used DHHC inhibitor in the field. Possessing an acrylamide warhead, PATi pairs its gain in potency with decreases in both toxicity and inhibition of the S-acylation eraser enzymes - two of the major weaknesses of 2BP. Our studies show that PATi engages with DHHC family proteins in cells, inhibits protein S-acylation, and disrupts DHHC-regulated cellular events. PATi represents an improved chemical tool for untangling the complexities of DHHC-mediated cell signaling by protein S-acylation.
\end{abstract}

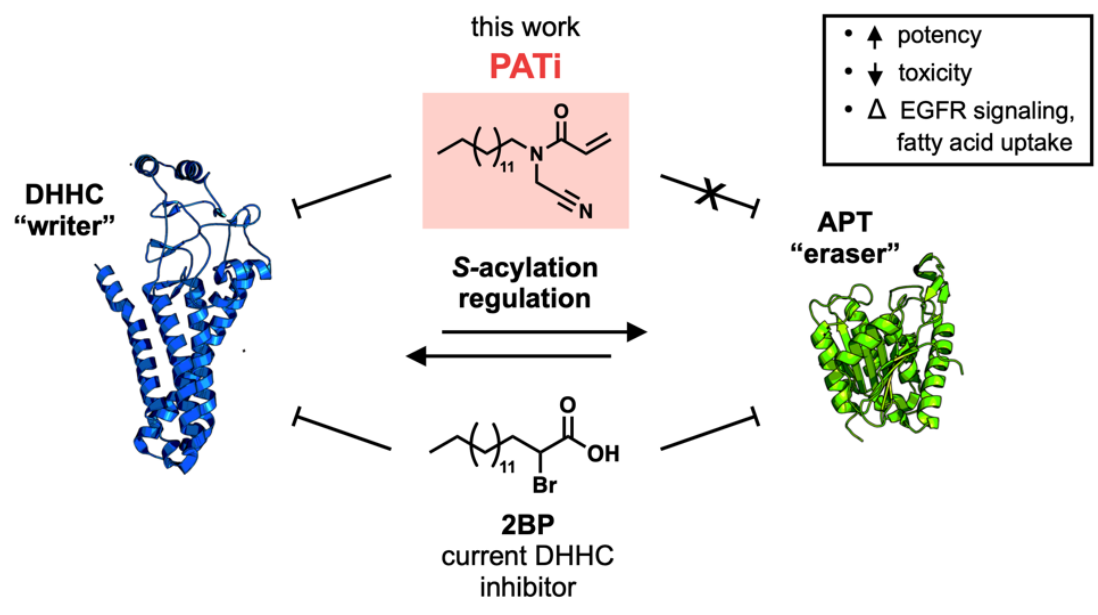




\section{Introduction}

Protein S-acylation is the post-translational addition of a long chain fatty acid to cysteine thiols via a thioester bond ${ }^{1-2}$ and is often referred to as $S$-palmitoylation due to the prevalence of C16:0 modification. S-acylation has complex and wide-ranging effects on target proteins; not only can it alter membrane association and subcellular trafficking, as for the well-studied oncogene $\operatorname{Ras}^{3}$, but it also regulates protein oligomerization, activity, and stability ${ }^{3-7}$. Sacylation is enzymatically reversible, with lipid addition catalyzed by the protein acyl transferases (PATs), which possess an active site Asp-His-His-Cys domain (earning them the moniker "DHHC") and lipid removal, by a suite of serine hydrolase family acyl-protein thioesterases (APTs), including APT1 ${ }^{8}, \mathrm{APT} 2{ }^{9}, \mathrm{ABHD} 10^{6}$, and $\mathrm{ABHD} 17 \mathrm{~A} / \mathrm{B} / \mathrm{C}{ }^{10}$. The activity of both these DHHC "writer" and APT "eraser" proteins is tightly regulated, and together, they in turn dynamically regulate protein S-acylation. Disruption of this cycle is consequential at the cellular and organismal levels ${ }^{11-13}$.

In particular, dysregulation of DHHC family proteins is associated with human pathology, including cancer progression, inflammation, and neurological dysfunction ${ }^{14-16}$. For example, zDHHC9 is upregulated in colorectal cancer and has been implicated in the pathogenesis of leukemia ${ }^{17-18}$, while its loss-of-function mutations are associated with X-linked intellectual disability (XLID) ${ }^{19-20}$. ZDHHC20 regulates the signaling of the receptor tyrosine kinase EGFR, and its activity has been implicated in cellular transformation and lung tumorigenesis ${ }^{21-22}$. Finally, knockdown of zDHHC7 has been shown to mitigate symptoms of inflammatory bowel disease, possibly via loss of STAT3 palmitoylation and activation ${ }^{23}$.

Despite the annotation of the first palmitoyl transferase in yeast two decades ago ${ }^{24-25}$, a paucity of tools to perturb the function of the DHHC family has hindered progress in our understanding of their mechanism, regulatory roles, and connections to disease states. Functional redundancy between many DHHCs, as well as inter-family regulation ${ }^{26-28}$, limit the use of classic genetic tools and highlight the necessity of small molecule inhibitors. While the $\alpha$-brominated fatty acid 2-bromopalmitate (2BP) is the most commonly used small molecule tool to study DHHC functions in live cells, its ubiquity obscures its promiscuity, poor utility, and cellular toxicity. 2BP acts as a pan-DHHC inhibitor by covalently modifying the active cysteine residues of DHHC proteins, either as 2BP or the 2BP-CoA adduct, with the latter metabolic product displaying enhanced reactivity with proteome ${ }^{29}$. In fact, 2BP inhibits at least two of the S-acylation erasers, APT1 and APT2, in cells ${ }^{30}$. Moreover, 2BP is often used at micromolar concentrations (10-100 
$\mu \mathrm{M})$, concentrations that are at or above its toxicity threshold ${ }^{27,29,31}$. Other reported $S$-acylation inhibitors, such as cerulenin, tunicamycin, and compound $V$, suffer from significant toxicity, poor selectively, and/or a lack of characterization in cells ${ }^{32-34}$. Thus, there is a critical need for new chemical probes to study DHHC-mediated S-acylation in endogenous contexts.

Here, we report the development of PATi, a covalent pan-DHHC inhibitor with an acrylamide warhead and improved properties relative to 2BP. Not only is PATi more potent than 2BP in vitro, it is also less toxic and does not inhibit APT1 or APT2, thereby addressing two key limitations of 2BP. We demonstrate that PATi inhibits S-acylation and engages directly with DHHC family proteins in cellulo. Finally, we show that PATi can modulate DHHC-dependent cellular effects, including EGFR-mediated cell signaling and CD36-mediated lipid uptake and droplet formation. Overall, this work introduces PATi as a chemical tool to complement current methods to study the biological implications of disrupting DHHC-mediated S-acylation.

\section{Acrylamide-based molecules inhibit zDHHC20}

The DHHC family proteins are thought to employ a two-step mechanism, in which the cysteine thiolate of the active site attacks the fatty acyl-CoA thioester, resulting in an autoacylated DHHC with the acyl chain bound in the hydrophobic groove. The fatty acyl chain is then transferred from the cysteine to a protein substrate. This nucleophilic cysteine can be targeted by electrophiles - like 2BP - to hamper DHHC activity. In pursuit of a still potent but less reactive inhibitor in cellulo, we proposed to exchange the a-halo carbonyl of 2BP with an acrylamide warhead. Acrylamides are known to react faster with cysteine thiols than serine alcohols, and, unlike the 2BP fatty acid, will likely not undergo metabolic conversion to reactive and nonspecific acyl CoA intermediates ${ }^{29}, 35$. Moreover, the acrylamide scaffold is achiral and readily synthetically accessible. Therefore, we designed and synthesized 1 (Figure 1A), which features a 14 carbon-long lipid tail appended to the acrylamide warhead. Docking of 1 on the only published crystal structure of a human DHHC (ZDHHC20) showed that the warhead is proximal $(3.5 \AA)$ to the active site Cys (Figure S1). We therefore tested 1 against purified zDHHC20 using a fluorescence polarization (FP) assay with a fluorophore-tagged peptide that we identified as a substrate of $z D H H C 20,5-F A M-N R a s$ (Figure S2). 1 ( IC $_{50}=21.4 \pm 5.9 \mu \mathrm{M}$ ) successfully inhibited $z \mathrm{DHHC} 20$, although with decreased activity as compared to $2 \mathrm{BP}\left(\mathrm{IC}_{50}=\right.$ $5.33 \pm 0.77 \mu \mathrm{M}$ ), confirming that an acrylamide-based lipid inhibitor can inhibit zDHHC20. Thus, we decided to explore the structure-activity relationship of 1 in an effort to enhance its potency. 


\section{PATi is a potent zDHHC20 inhibitor}

To optimize 1, we designed and synthesized a panel of compounds, all with an acrylamide appended to a lipid tail. First, we targeted the lipid tail, shortening it by four carbons (2). This change abrogated the zDHHC20 inhibitory properties entirely, demonstrating the criticality of extensive contacts with the hydrophobic channel and inspiring us to instead focus on modifying the acrylamide moiety. We next tested compounds with either a substituted acrylamide amine and/or a butenoic acid-modified amide (Figure 1B). While most performed worse than the parent molecule, one molecule functionalized with a cyanomethyl group, PATi, showed significantly improved activity against zDHHC20, with approximately $80 \%$ inhibition at $10 \mu \mathrm{M}$. PATi was found to have an $\mathrm{IC}_{50}$ of $1.35 \pm 0.26 \mu \mathrm{M}$ against $\mathrm{zDHHC} 20$, a 5 -fold improvement over 2BP (Figure S3). Moreover, addition of the cyanomethyl group to the cis butenoic acid derivative (3) improved its activity (12), highlighting the criticality of this moiety. A second molecule, 8, which possesses a terminal alkyne in lieu of the cyanomethyl, was similar in potency to $2 \mathrm{BP}$, with an $\mathrm{IC}_{50}$ of $8.32 \pm 2.25 \mu \mathrm{M}$ (Figure S3). To confirm that the reactivity of PATi and 8 stemmed from the acrylamide warhead and not reversible thioimidate complex formation, we synthesized and tested 13 and 14, the alkyl amide analogues of 8 and PATi, respectively (Figure 1B). Both molecules were inactive, verifying the acrylamide as the DHHCreactive moiety. In sum, PATi is a potent $z \mathrm{DHHC} 20$ inhibitor in vitro and requires both the cyanomethyl group and acrylamide warhead for activity. Therefore, to assess its utility in live cells, we next assessed its inhibition of APTs and toxicity.

\section{PATi does not inhibit APTs and is less toxic than 2BP}

To determine if PATi inhibits eraser APTs, as observed with 2BP, we evaluated the effects of PATi relative to $2 \mathrm{BP}$ on $\mathrm{APT} 1$ and $\mathrm{APT} 2$ activity in vitro using a fluorogenic probe for APT activity, DPP ${ }^{36}$. PATi showed little inhibition of APT1 and APT2, while 2BP, in agreement with previous reports, significantly inhibited both APT1 and APT2 (Figure 2A) ${ }^{30}$. At $25 \mu \mathrm{M}$, a concentration at or below which 2BP is often used, 2BP abolished most activity of APT1 and all activity of APT2. In striking contrast, PATi showed no inhibition of APT1 or APT2 even at $50 \mu \mathrm{M}$, the highest concentration assayed. This data suggests that PATi, unlike 2BP, can be used to monitor the dynamics and the kinetics of acylation without perturbing APT-mediated deacylation.

We also compared the toxicity of PATi to that of 2BP across a panel of commonly used mammalian cell lines, including MDA-MB-231, HEK293T, HepG2, and 3T3-L1. After 6 hours of 
treatment, PATi's toxicity was limited up to $100 \mu \mathrm{M}$, while 2BP significantly reduced cell viability at much lower concentrations (Figure 2B, S4). For example, in MDA-MB-231 cells, treatment with 2BP resulted in $\sim 80 \%$ cell death at $40 \mu \mathrm{M}$, whereas at the same concentration of PATi, no toxicity was observed. As PATi was non-toxic at $20 \mu \mathrm{M}$ across all cell lines we tested, we designated this as our maximum concentration of PATi to use for assessment in cells. Overall, these results - the lack of APT inhibition and decreased toxicity - paired with the robust inhibition of zDHHC20 in vitro, suggested that PATi might be an inhibitor of zDHHC-mediated acylation in cells.

\section{PATi inhibits protein S-acylation in live cells}

To validate the use of PATi as an inhibitor of S-acylation, we next sought to assess its efficacy and potency in live cells. Using 17-octadecynoic acid (17-ODYA) metabolic labeling, wherein a clickable fatty acid is used to monitor lipid incorporation, we observed a dose-dependent decrease in global S-acylation when cells were treated with PATi (Figure 2C, S5) ${ }^{37}$. We further confirmed the global inhibition of protein S-acylation by PATi using acyl-biotin exchange (ABE) ${ }^{38}$, a method for the enrichment and visualization of acylated cysteine residues (Figure 2D).

To corroborate these proteome-wide observations, we next tested whether PATi could inhibit the S-acylation of several well-studied DHHC substrate proteins. We overexpressed either the human immune adaptor protein Myd88 or Legionella E3 ligase GobX, whose S-acylation is regulated by zDHHC6 and zDHHC20, respectively, in HEK293T cells, and then treated the cells with a range of concentrations of PATi ${ }^{39-40}$. Assessment of these exogenous substrates using ABE revealed a dose-dependent decrease in S-acylation, with GobX displaying greater sensitivity to PATi than Myd88 (Figure 2E, F). Furthermore, in both of these ABE assays, we also observed a dose-dependent decrease in the S-acylation of endogenous Ras upon treatment with PATi. All targets displayed reduced acylation at a concentration of $20 \mu \mathrm{M} \mathrm{PATi}$, establishing this as our working concentration. Collectively, these data confirm that PATi inhibits protein S-acylation in live cells.

\section{PATi engages DHHCs in live cells}

Having verified that PATi inhibits zDHHC20 in vitro and blocks protein S-acylation in live cells, we next sought to confirm direct engagement of DHHC family proteins by PATi in live cells. We postulated that 8, an analogue of PATi possessing a terminal alkyne instead of the cyanomethyl group, could be used to visualize PATi engagement across the DHHC family by reporter 
conjugation via copper-catalyzed azide-alkyne "click" cycloaddition (Figure 1A). Therefore, we first determined which DHHCs could be labeled by 8 . HEK293T cells overexpressing individual DHHCs were treated with $\mathbf{8}$, and, following cell lysis, subjected to a click reaction with TAMRAazide in order to label 8-modified proteins. In-gel fluorescence indicated that 8 labels ZDHHC2, $6,9,13,14,15,18,20$, and 24 , all of the DHHCs in our library with strong expression (Figure S6). Furthermore, 8 was also found to label endogenous DHHC proteins, including zDHHC5, which was not labeled in the fluorescence screen, likely due to poor expression (Figure S7).

We next examined the residues of DHHC proteins labelled by $\mathbf{8}$. As the cysteine residue within the DHHC motif is critical for the $S$-acyltransferase activity for DHHC proteins $24-25,41-42$, we performed 8 labeling on a subset of exogenous catalytically dead (DHHS) mutants. While some DHHCs, such as ZDHHC9 and 24, evinced decreased labeling of the active site mutant, other $\mathrm{DHHCs}$, including zDHHC20, did not, suggesting that the DHHC motif Cys is not the only residue modified by $\mathbf{8}$, as is also observed with 16C-BYA, an alkyne-containing analogue of 2BP ${ }^{43}$ (Figure S8). Finally, we attempted to use mass spectrometry (MS) in order to see if 8 could label more DHHCs than observed via gel-based methods. To this end, following treatment of HEK293T cells with 8 or DMSO, click reaction with biotin-azide, enrichment, and on-bead trypsin digestion, digested peptides were identified and quantified by dimethyl labeling. In our dataset, endogenous zDHHC6 - a DHHC not identified in our gel-based assays - was identified as a high-confidence target in two independent replicates (Figure S9). No other DHHC proteins were identified in our dataset, likely due to their low abundance and hydrophobicity, which complicate MS analysis ${ }^{29,43-45}$.

Having validated 8's ability to label DHHC family proteins, we next performed competitive labeling with PATi for all exogenous (zDHHC2, zDHHC9, zDHHC14, zDHHC15, and zDHHC24) and endogenous (zDHHC5, 13, and 18) DHHC proteins labeled by 8 in order to confirm PATi target engagement in live cells. For the exogenous DHHCs, cells overexpressing each targeted protein were treated with PATi $(0,10$, and $20 \mu \mathrm{M}, 2$ hours), chased with 8 ( $1 \mu \mathrm{M}, 2$ hours), and then lysed. After TAMRA-azide ligation, in-gel fluorescence indicated that PATi blocked 8 labeling, confirming that PATi engages each of these DHHC targets (Figure 3A). To probe the endogenous DHHCs, we again performed competitive labeling, this time on non-transfected HEK293T cells and using 8 at $20 \mu \mathrm{M}$ for 3 hours for the chase. Here, after conjugation with biotin-azide, pulldown, and Western blotting, we observed that PATi impeded the 8 labeling of zDHHC5, zDHHC18, and two isoforms of zDHHC13 (Figure 3B) - confirming PATi directly 
engages with these DHHCs as well. The ability of PATi to outcompete 8 labeling of both exogenous and endogenous DHHCs suggests that PATi inhibition of S-acylation in live cells likely stems from inhibition of DHHC family proteins.

\section{PATi inhibits the $S$-acylation of EGFR and CD36}

Finally, we sought to test whether PATi could recapitulate observations reported to stem from DHHC disruption and establish that, along with on-target binding, PATi can be used to discern cellular outcomes from loss of $S$-acylation. A critical $S$-acylated protein and substrate of zDHHC20 is the epidermal growth factor receptor (EGFR), a receptor tyrosine kinase whose activity is widely dysregulated in cancer ${ }^{21}$. There are several mechanisms by which its Cterminal acylation regulates its activity; in a KRAS-mutant context, loss of its palmitoylation is reported to disrupt signaling along the phosphatidylinositol 3-kinase (PI3K) pathway ${ }^{22}$. Thus, we first confirmed that PATi diminished EGFR S-acylation in MDA-MB-231 cancer cells at its working concentration (Figure 4A) and then assessed whether PATi-mediated interruption of EGFR acylation impacted downstream PI3K pathway signaling. We observed that treatment with PATi moderated the phosphorylation of protein kinase $B$ (Akt) and the abundance of the transcription factor MYC, two markers of EGFR-P13K pathway activity (Figure 4B, S10). These results paralleled those seen with genetic knockdown of ZDHHC20 and established PATi's ability to validate results downstream of acylation disruption ${ }^{22}$.

To further substantiate PATi's ability to modulate DHHC-dependent cellular functions, we next evaluated whether PATi could inhibit the S-acylation and subsequently the activity of CD36. CD36 is an immuno-metabolic glycoprotein and a substrate of zDHHC4 and zDHHC5, two DHHCs we confirmed were targeted by PATi (Figure 3B, S11). The S-acylation of CD36 mediates its localization and in turn its fatty acid translocase activity ${ }^{46}$. We found that treatment of 3T3-L1 pre-adipocytes with PATi not only reduced CD36 acylation levels (Figure 4C), but also decreased the uptake of fatty acids and formation of lipid droplets, results previously observed with knockdown of zDHHC4/5 (Figure 4D, S12). Together, these results indicate that not only does PATi perturb the acylation of significant endogenous targets, it also modulates their known acylation-dependent activity and functionality.

\section{Conclusions}

As our understanding of the role of protein S-acylation in regulating cell and organismal biology grows, the toxicity and limited potency of current inhibitors demand improved chemical tools. In 
this Article, we introduced PATi, an inhibitor of DHHC family proteins, to complement current methods to probe $S$-acylation and its consequences in cells. Featuring an acrylamide warhead functionalized with a cyanomethyl group, PATi is more potent than 2BP - the leading inhibitor in the field - and, more importantly, has a significantly improved toxicity profile across multiple mammalian cells lines. We also show that PATi does not inhibit eraser APTs, a critical limitation of 2BP. These improvements were achieved without a loss of in-cell efficacy; for example, PATi inhibited the acylation of CD36 at $20 \mu \mathrm{M}$, while $2 \mathrm{BP}$ was used at $100 \mu \mathrm{M}{ }^{46}$. Furthermore, we used 8, an alkyne-containing derivative of PATi, to show that PATi engages with DHHC family proteins across clades, validating PATi as a pan-DHHC inhibitor and confirming its role in perturbing DHHC-dependent acylation. Finally, we applied PATi to assess cellular responses previously reported to result from loss of $S$-acylation. We demonstrated that decreases in the acylation of EGFR and CD36 caused by PATi treatment were paired with changes in signaling activity and lipid uptake, respectively - changes aligned with results reported using both genetic manipulation and 2BP treatment ${ }^{21,27}$. These results not only emphasize the importance of $S$ acylation in biological processes, but also highlight the potential of PATi in elucidating the regulatory roles of $\mathrm{DHHC}$ family proteins.

While this work reveals the promise of acrylamide based DHHC family inhibitors, it also underscores the need for additional improvements in selectivity and potency. MS analysis suggests that 8 can label $\sim 396$ proteins with high confidence in cells, over $75 \%$ of which are annotated as acylated proteins (Table S1, Figure S9). These results suggest that $\mathbf{8}$, and in turn PATi, interact with acylated targets and emphasize the disadvantage of their lipid "tails." As mimetics of fatty acids and their derivatives, aliphatic chains are thought to lead to numerous off-targets in cells ${ }^{47}$. Therefore, modification or replacement of the lipid will be critical in the next generation of molecules. As comparison of 1 with 2 indicates, though, the loss of hydrophobic contacts would need to be compensated for by expansion of the scaffold and increased contacts with the protein elsewhere. Interestingly, we observed that the cyanomethyl group was crucial for PATi potency; however, the reasons for its criticality are unclear. We envision that a crystal structure of a PATi-bound DHHC would both aid in deciphering the mechanism of the cyanomethyl-mediated increase in potency and highlight opportunities for structure-based design. In addition, the capability of $\mathrm{zDHHC} 20$ to acylate NRas peptide, as well as recent reports of DHHC-targeted peptide inhibitors, hint at the potential of peptide scaffolds as DHHC inhibitors ${ }^{48-49}$. Our future work will focus on modifying small molecule and peptide scaffolds to achieve improvements in selectivity and potency. 


\section{Acknowledgements}

This research was supported by the University of Chicago, the National Institute of General Medical Sciences of the National Institutes of Health NIH (R35 GM119840, to B.C.D.), the National Institute of Diabetes and Digestive and Kidney Diseases of the National Institutes of Health (F30 DK125088, to S-A.A.), and the Swiss National Science Foundation (P2BEP2_188250, to C.D.). We thank the Mass Spectrometry Core facility at University of Illinois at Chicago for providing technical advice on sample preparation for proteomics. We thank S. Bamji (University of British Columbia), M. Fukata (NIPS, Japan), M. Machner (NIH) and B. Beutler (University of Texas Southwestern Medical Center) for providing the various plasmids used in this work.

\section{Notes}

R.S.K. and B.C.D. have a patent on the DPP technology used in this work.

\section{Supporting Information}

Supporting figures, tables, spectra, procedures, and analysis 
A
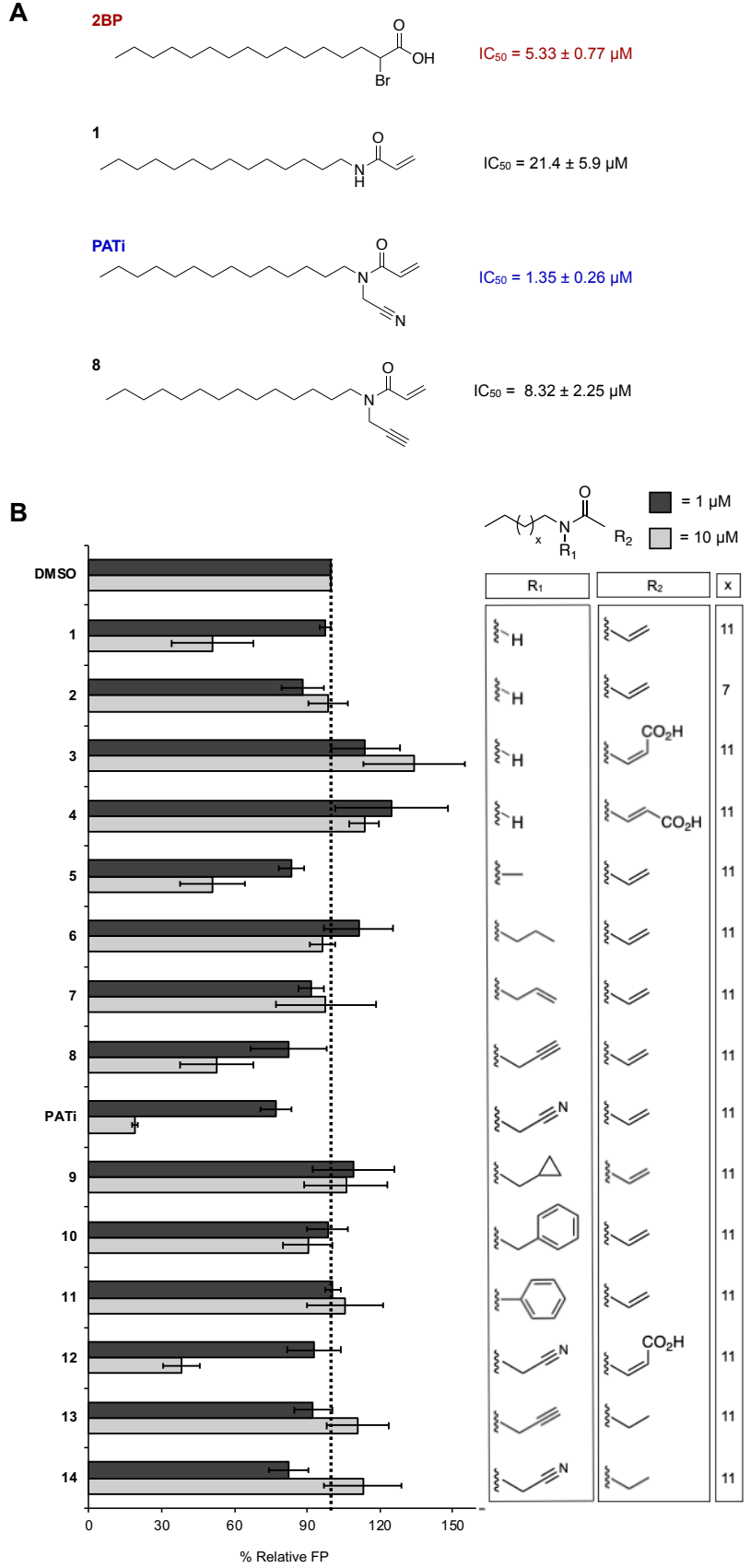

Figure 1. Discovery of and in vitro inhibition data against human zDHHC20 for PATi. (A) Structure and $\mathrm{IC}_{50}$ against human $\mathrm{zDHHC} 20$ of key molecules used in this work. (B) Fluorescence polarization (FP) screening of a panel of acrylamide-based molecules against zDHHC20, with activity normalized to DMSO. Data are presented as the mean \pm standard deviation $(n=3)$. 
A

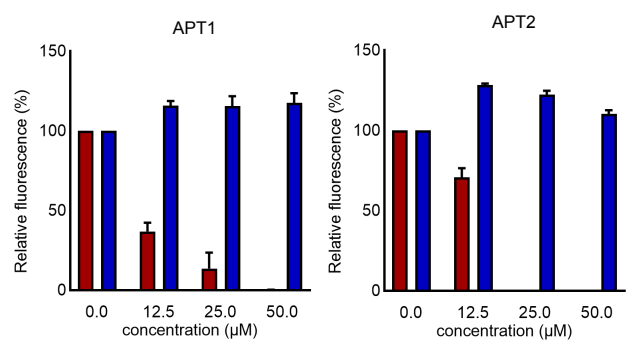

B
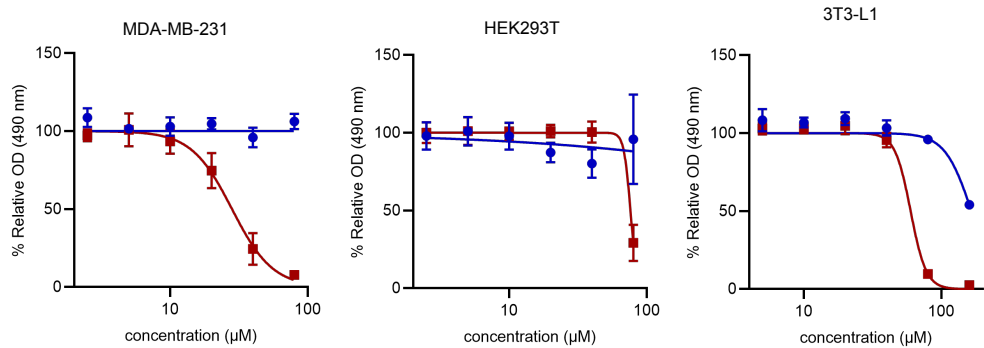

C

D
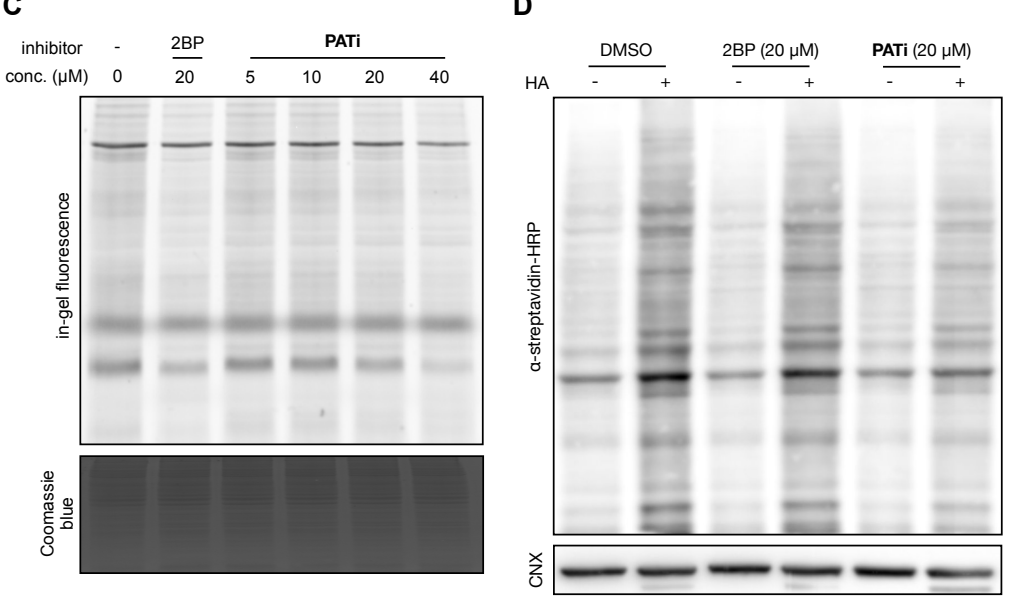

E

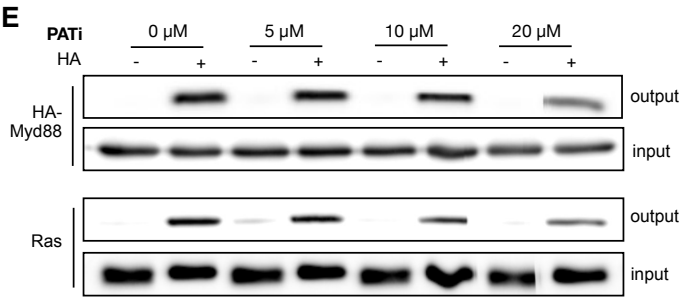

$\mathbf{F}$

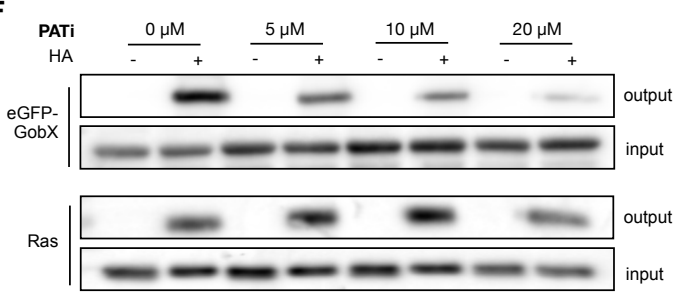

Figure 2. Validation and characterization of PATi in vitro and in cellulo. (A) Incubation of purified APT1 or APT2 (50 nM) with either 2BP or PATi, followed by measurement of APT activity using the fluorogenic probe DPP-5. Data are presented as the mean \pm standard deviation ( $n=3$ ). (B) Viability of HEK293T, MDA-MB-231, and 3T3-L1 cells treated with varied concentrations of either 2BP or PATi ( 6 hours), as measured by MTS assay. Data are presented as the mean \pm standard deviation $(n \geq 3)$. (C) HEK293T cells pretreated with DMSO, 2BP $(20 \mu \mathrm{M}, 1$ hour), or PATi $(0,5,10,20$, and $40 \mu \mathrm{M}, 1$ hour) before treatment with 17octadecynoic acid (17-ODYA) (6 hours) to metabolically label palmitoylated proteins. Isolated proteomes were then subject to click chemistry to conjugate a fluorophore on proteins modified by 17-ODYA, followed by protein separation via SDS-PAGE. In-gel fluorescence revealed proteome-wide inhibition of protein palmitoylation by PATi. (D) Acyl-biotin exchange (ABE) of HEK293T cells treated with 2BP or PATi $(20 \mu \mathrm{M}, 6$ hours). Global S-acylation visualized using streptavidin-HRP. (E,F) Dose-response change in the $S$-acylation of exogenous HA-tagged Myd88 or eGFP-tagged GobX and endogenous Ras in HEK293T cells upon PATi treatment (6 hours) as measured by ABE. 
A
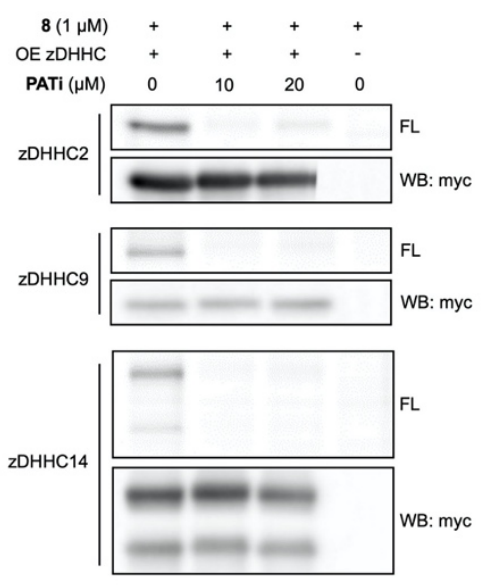
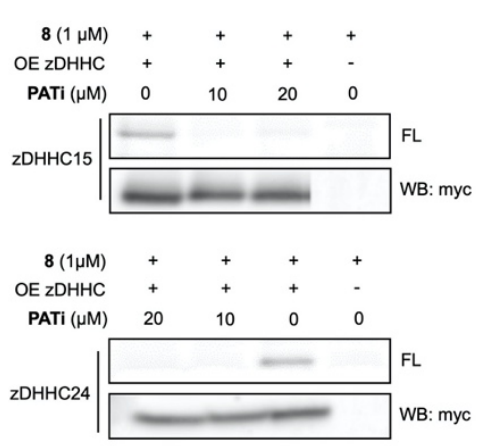

B

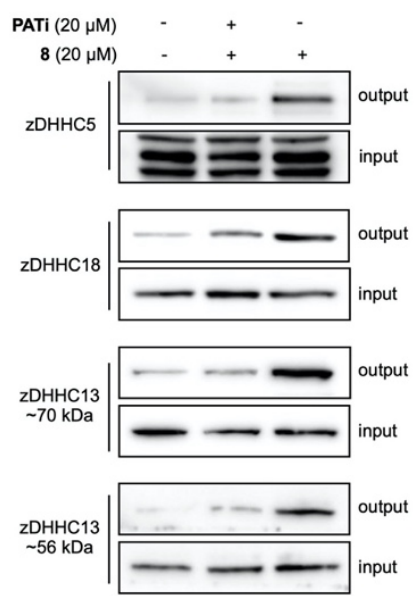

Figure 3. Engagement of PATi with DHHC family proteins. (A) In-gel fluorescence analysis of myc-tagged DHHC proteins overexpressed in HEK293T cells treated with PATi or DMSO (2 hours), followed by labeling with 8 ( $1 \mu \mathrm{M}, 2$ hours). Expression levels visualized via anti-myc tag Western blot. (B) Western blot analysis of endogenous DHHC proteins in HEK293T cells treated with PATi or DMSO (3 hours), followed by labeling with 8 (20 $\mu \mathrm{M}, 3$ hours). 
A

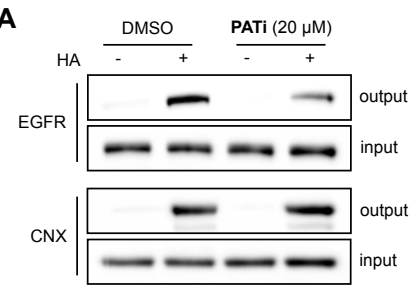

C

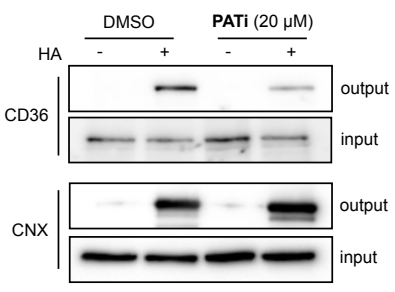

B

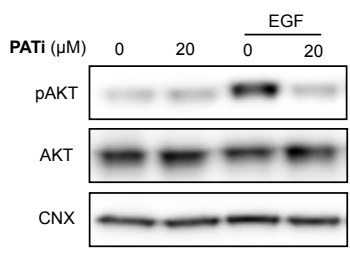

D

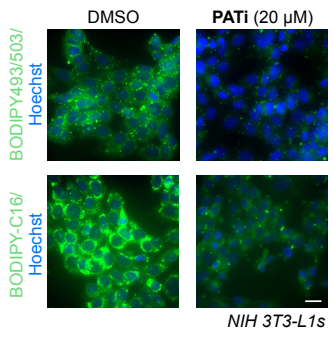

Figure 4. Confirmation of PATi functional activity in cellulo. (A) Analysis of EGFR S-acylation via $A B E$ upon PATi treatment $(20 \mu \mathrm{M}, 6$ hours) in MDA-MB-231 cells. (B) EGF (100 ng/mL, 15 minutes)-induced phosphorylation of AKT with and without PATi treatment $(20 \mu \mathrm{M}, 3$ hours). (C) Analysis of CD36 S-acylation via ABE upon PATi treatment $(20 \mu \mathrm{M}, 6$ hours $)$ in 3T3-L1 cells. (D) Representative images of fluorescence imaging of 3T3-L1 preadipocytes starved (12 hours) and treated with DMSO or PATi $(20 \mu \mathrm{M}, 3$ hours), followed by $10 \mu \mathrm{M}$ BSA-bound oleate and BODIPY493/503 or BODIPY-palmitate $(2 \mu \mathrm{M}, 3$ hours) $(n=5)$. Scale bar $=20 \mu \mathrm{M}$. 


\section{References}

1. Blanc, M.; David, F.; Abrami, L.; Migliozzi, D.; Armand, F.; Burgi, J.; van der Goot, F. G., SwissPalm: Protein Palmitoylation database. F1000Res 2015, 4, 261.

2. Lanyon-Hogg, T.; Faronato, M.; Serwa, R. A.; Tate, E. W., Dynamic Protein Acylation: New Substrates, Mechanisms, and Drug Targets. Trends Biochem Sci 2017, 42 (7), 566-581.

3. Linder, M. E.; Deschenes, R. J., Palmitoylation: policing protein stability and traffic. Nat Rev Mol Cell Biol 2007, 8 (1), 74-84.

4. Bhattacharyya, R.; Fenn, R. H.; Barren, C.; Tanzi, R. E.; Kovacs, D. M., Palmitoylated APP Forms Dimers, Cleaved by BACE1. PLoS One 2016, 11 (11), e0166400.

5. Seno, K.; Hayashi, F., Palmitoylation is a prerequisite for dimerization-dependent raftophilicity of rhodopsin. J Biol Chem 2017, 292 (37), 15321-15328.

6. Cao, Y.; Qiu, T.; Kathayat, R. S.; Azizi, S. A.; Thorne, A. K.; Ahn, D.; Fukata, Y.; Fukata, M.; Rice, P. A.; Dickinson, B. C., ABHD10 is an S-depalmitoylase affecting redox homeostasis through peroxiredoxin-5. Nat Chem Biol 2019, 15 (12), 1232-1240.

7. Bolland, D. E.; Moritz, A. E.; Stanislowski, D. J.; Vaughan, R. A.; Foster, J. D., Palmitoylation by Multiple DHHC Enzymes Enhances Dopamine Transporter Function and Stability. ACS Chem Neurosci 2019, 10 (6), 2707-2717.

8. Duncan, J. A.; Gilman, A. G., A cytoplasmic acyl-protein thioesterase that removes palmitate from G protein alpha subunits and p21(RAS). J Biol Chem 1998, 273 (25), 15830-7.

9. Tomatis, V. M.; Trenchi, A.; Gomez, G. A.; Daniotti, J. L., Acyl-protein thioesterase 2 catalyzes the deacylation of peripheral membrane-associated GAP-43. PLoS One 2010, 5 (11), e15045.

10. Lin, D. T.; Conibear, E., ABHD17 proteins are novel protein depalmitoylases that regulate N-Ras palmitate turnover and subcellular localization. Elife 2015, 4, e11306.

11. Ladygina, N.; Martin, B. R.; Altman, A., Dynamic palmitoylation and the role of DHHC proteins in T cell activation and anergy. Adv Immunol 2011, 109, 1-44.

12. Jiang, H.; Zhang, X.; Chen, X.; Aramsangtienchai, P.; Tong, Z.; Lin, H., Protein Lipidation: Occurrence, Mechanisms, Biological Functions, and Enabling Technologies. Chem Rev 2018, 118 (3), 919-988.

13. Gok, C.; Fuller, W., Topical review: Shedding light on molecular and cellular consequences of NCX1 palmitoylation. Cell Signal 2020, 76, 109791.

14. Chen, X.; Ma, H.; Wang, Z.; Zhang, S.; Yang, H.; Fang, Z., EZH2 Palmitoylation Mediated by ZDHHC5 in p53-Mutant Glioma Drives Malignant Development and Progression. Cancer Res 2017, 77 (18), 4998-5010.

15. Beard, R. S., Jr.; Yang, X.; Meegan, J. E.; Overstreet, J. W.; Yang, C. G.; Elliott, J. A.; Reynolds, J. J.; Cha, B. J.; Pivetti, C. D.; Mitchell, D. A.; Wu, M. H.; Deschenes, R. J.; Yuan, S. Y., Palmitoyl acyltransferase DHHC21 mediates endothelial dysfunction in systemic inflammatory response syndrome. Nat Commun 2016, 7, 12823.

16. Mukai, J.; Liu, H.; Burt, R. A.; Swor, D. E.; Lai, W. S.; Karayiorgou, M.; Gogos, J. A., Evidence that the gene encoding ZDHHC8 contributes to the risk of schizophrenia. Nat Genet 2004, 36 (7), 72531.

17. Young, E.; Zheng, Z. Y.; Wilkins, A. D.; Jeong, H. T.; Li, M.; Lichtarge, O.; Chang, E. C., Regulation of Ras localization and cell transformation by evolutionarily conserved palmitoyltransferases. $\mathrm{Mol}$ Cell Biol 2014, 34 (3), 374-85.

18. Liu, P.; Jiao, B.; Zhang, R.; Zhao, H.; Zhang, C.; Wu, M.; Li, D.; Zhao, X.; Qiu, Q.; Li, J.; Ren, R., Palmitoylacyltransferase Zdhhc9 inactivation mitigates leukemogenic potential of oncogenic Nras. Leukemia 2016, 30 (5), 1225-8.

19. Raymond, F. L.; Tarpey, P. S.; Edkins, S.; Tofts, C.; O'Meara, S.; Teague, J.; Butler, A.; Stevens, C.; Barthorpe, S.; Buck, G.; Cole, J.; Dicks, E.; Gray, K.; Halliday, K.; Hills, K.; Hinton, J.; Jones, D.; Menzies, A.; Perry, J.; Raine, K.; Shepherd, R.; Small, A.; Varian, J.; Widaa, S.; Mallya, U.; Moon, J.; Luo, Y.; Shaw, M.; Boyle, J.; Kerr, B.; Turner, G.; Quarrell, O.; Cole, T.; Easton, D. F.; Wooster, R.; Bobrow, M.; Schwartz, C. E.; Gecz, J.; Stratton, M. R.; Futreal, P. A., Mutations in ZDHHC9, which 
encodes a palmitoyltransferase of NRAS and HRAS, cause X-linked mental retardation associated with a Marfanoid habitus. Am J Hum Genet 2007, 80 (5), 982-7.

20. Tarpey, P. S.; Smith, R.; Pleasance, E.; Whibley, A.; Edkins, S.; Hardy, C.; O'Meara, S.; Latimer, C.; Dicks, E.; Menzies, A.; Stephens, P.; Blow, M.; Greenman, C.; Xue, Y.; Tyler-Smith, C.; Thompson, D.; Gray, K.; Andrews, J.; Barthorpe, S.; Buck, G.; Cole, J.; Dunmore, R.; Jones, D.; Maddison, M.; Mironenko, T.; Turner, R.; Turrell, K.; Varian, J.; West, S.; Widaa, S.; Wray, P.; Teague, J.; Butler, A.; Jenkinson, A.; Jia, M.; Richardson, D.; Shepherd, R.; Wooster, R.; Tejada, M. I.; Martinez, F.; Carvill, G.; Goliath, R.; de Brouwer, A. P.; van Bokhoven, H.; Van Esch, H.; Chelly, J.; Raynaud, M.; Ropers, H. H.; Abidi, F. E.; Srivastava, A. K.; Cox, J.; Luo, Y.; Mallya, U.; Moon, J.; Parnau, J.; Mohammed, S.; Tolmie, J. L.; Shoubridge, C.; Corbett, M.; Gardner, A.; Haan, E.; Rujirabanjerd, S.; Shaw, M.; Vandeleur, L.; Fullston, T.; Easton, D. F.; Boyle, J.; Partington, M.; Hackett, A.; Field, M.; Skinner, C.; Stevenson, R. E.; Bobrow, M.; Turner, G.; Schwartz, C. E.; Gecz, J.; Raymond, F. L.; Futreal, P. A.; Stratton, M. R., A systematic, large-scale resequencing screen of X-chromosome coding exons in mental retardation. Nat Genet 2009, 41 (5), 535-43.

21. Runkle, K. B.; Kharbanda, A.; Stypulkowski, E.; Cao, X. J.; Wang, W.; Garcia, B. A.; Witze, E. S., Inhibition of DHHC20-Mediated EGFR Palmitoylation Creates a Dependence on EGFR Signaling. Mol Cell 2016, 62 (3), 385-396.

22. Kharbanda, A.; Walter, D. M.; Gudiel, A. A.; Schek, N.; Feldser, D. M.; Witze, E. S., Blocking EGFR palmitoylation suppresses PI3K signaling and mutant KRAS lung tumorigenesis. Sci Signal 2020, 13 (621).

23. Sanders, S. S.; Hayden, M. R., Aberrant palmitoylation in Huntington disease. Biochem Soc Trans 2015, 43 (2), 205-10.

24. Roth, A. F.; Feng, Y.; Chen, L.; Davis, N. G., The yeast DHHC cysteine-rich domain protein Akr1p is a palmitoyl transferase. J Cell Biol 2002, 159 (1), 23-8.

25. Lobo, S.; Greentree, W. K.; Linder, M. E.; Deschenes, R. J., Identification of a Ras palmitoyltransferase in Saccharomyces cerevisiae. J Biol Chem 2002, 277 (43), 41268-73.

26. Fukata, M.; Fukata, Y.; Adesnik, H.; Nicoll, R. A.; Bredt, D. S., Identification of PSD-95 palmitoylating enzymes. Neuron 2004, 44 (6), 987-96.

27. Wang, J.; Hao, J. W.; Wang, X.; Guo, H.; Sun, H. H.; Lai, X. Y.; Liu, L. Y.; Zhu, M.; Wang, H. Y.; Li, Y. F.; Yu, L. Y.; Xie, C.; Wang, H. R.; Mo, W.; Zhou, H. M.; Chen, S.; Liang, G.; Zhao, T. J., DHHC4 and DHHC5 Facilitate Fatty Acid Uptake by Palmitoylating and Targeting CD36 to the Plasma Membrane. Cell Rep 2019, 26 (1), 209-221 e5.

28. Abrami, L.; Dallavilla, T.; Sandoz, P. A.; Demir, M.; Kunz, B.; Savoglidis, G.; Hatzimanikatis, V.; van der Goot, F. G., Identification and dynamics of the human ZDHHC16-ZDHHC6 palmitoylation cascade. Elife 2017, 6.

29. Davda, D.; El Azzouny, M. A.; Tom, C. T.; Hernandez, J. L.; Majmudar, J. D.; Kennedy, R. T.; Martin, B. R., Profiling targets of the irreversible palmitoylation inhibitor 2-bromopalmitate. ACS Chem Biol 2013, 8 (9), 1912-7.

30. Pedro, M. P.; Vilcaes, A. A.; Tomatis, V. M.; Oliveira, R. G.; Gomez, G. A.; Daniotti, J. L., 2Bromopalmitate reduces protein deacylation by inhibition of acyl-protein thioesterase enzymatic activities. PLoS One 2013, 8 (10), e75232.

31. Tian, L.; McClafferty, H.; Jeffries, O.; Shipston, M. J., Multiple palmitoyltransferases are required for palmitoylation-dependent regulation of large conductance calcium- and voltage-activated potassium channels. J Biol Chem 2010, 285 (31), 23954-62.

32. Zheng, B.; Zhu, S.; Wu, X., Clickable analogue of cerulenin as chemical probe to explore protein palmitoylation. ACS Chem Biol 2015, 10 (1), 115-21.

33. DeJesus, G.; Bizzozero, O. A., Effect of 2-fluoropalmitate, cerulenin and tunicamycin on the palmitoylation and intracellular translocation of myelin proteolipid protein. Neurochem Res 2002, 27 (12), 1669-75.

34. Jennings, B. C.; Nadolski, M. J.; Ling, Y.; Baker, M. B.; Harrison, M. L.; Deschenes, R. J.; Linder, M. E., 2-Bromopalmitate and 2-(2-hydroxy-5-nitro-benzylidene)-benzo[b]thiophen-3-one inhibit DHHCmediated palmitoylation in vitro. $J$ Lipid Res 2009, 50 (2), 233-42. 
35. Jackson, P. A.; Widen, J. C.; Harki, D. A.; Brummond, K. M., Covalent Modifiers: A Chemical Perspective on the Reactivity of alpha,beta-Unsaturated Carbonyls with Thiols via Hetero-Michael Addition Reactions. J Med Chem 2017, 60 (3), 839-885.

36. Qiu, T.; Kathayat, R. S.; Cao, Y.; Beck, M. W.; Dickinson, B. C., A Fluorescent Probe with Improved Water Solubility Permits the Analysis of Protein S-Depalmitoylation Activity in Live Cells. Biochemistry 2018, 57 (2), 221-225.

37. Martin, B. R.; Cravatt, B. F., Large-scale profiling of protein palmitoylation in mammalian cells. Nat Methods 2009, 6 (2), 135-8.

38. Drisdel, R. C.; Green, W. N., Labeling and quantifying sites of protein palmitoylation. Biotechniques 2004, 36 (2), 276-85.

39. Kim, Y. C.; Lee, S. E.; Kim, S. K.; Jang, H. D.; Hwang, I.; Jin, S.; Hong, E. B.; Jang, K. S.; Kim, H. S., Toll-like receptor mediated inflammation requires FASN-dependent MYD88 palmitoylation. Nat Chem Biol 2019, 15 (9), 907-916.

40. Lin, Y. H.; Doms, A. G.; Cheng, E.; Kim, B.; Evans, T. R.; Machner, M. P., Host Cell-catalyzed SPalmitoylation Mediates Golgi Targeting of the Legionella Ubiquitin Ligase GobX. J Biol Chem 2015, 290 (42), 25766-81.

41. Jennings, B. C.; Linder, M. E., DHHC protein S-acyltransferases use similar ping-pong kinetic mechanisms but display different acyl-CoA specificities. J Biol Chem 2012, 287 (10), 7236-45.

42. Mitchell, D. A.; Mitchell, G.; Ling, Y.; Budde, C.; Deschenes, R. J., Mutational analysis of Saccharomyces cerevisiae Erf2 reveals a two-step reaction mechanism for protein palmitoylation by DHHC enzymes. J Biol Chem 2010, 285 (49), 38104-14.

43. Zheng, B.; DeRan, M.; Li, X.; Liao, X.; Fukata, M.; Wu, X., 2-Bromopalmitate analogues as activitybased probes to explore palmitoyl acyltransferases. J Am Chem Soc 2013, 135 (19), 7082-5.

44. Bastin, G.; Dissanayake, K.; Langburt, D.; Tam, A. L. C.; Lee, S. H.; Lachhar, K.; Heximer, S. P., RGS4 controls Galphai3-mediated regulation of Bcl-2 phosphorylation on TGN38-containing intracellular membranes. J Cell Sci 2020, 133 (12).

45. Zhang, Y.; Lin, Z.; Tan, Y.; Bu, F.; Hao, P.; Zhang, K.; Yang, H.; Liu, S.; Ren, Y., Exploration of Missing Proteins by a Combination Approach to Enrich the Low-Abundance Hydrophobic Proteins from Four Cancer Cell Lines. J Proteome Res 2020, 19 (1), 401-408.

46. Hao, J. W.; Wang, J.; Guo, H.; Zhao, Y. Y.; Sun, H. H.; Li, Y. F.; Lai, X. Y.; Zhao, N.; Wang, X.; Xie, C.; Hong, L.; Huang, X.; Wang, H. R.; Li, C. B.; Liang, B.; Chen, S.; Zhao, T. J., CD36 facilitates fatty acid uptake by dynamic palmitoylation-regulated endocytosis. Nat Commun 2020, 11 (1), 4765.

47. Arnott, J. A.; Planey, S. L., The influence of lipophilicity in drug discovery and design. Expert Opin Drug Discov 2012, 7 (10), 863-75.

48. Yao, H.; Lan, J.; Li, C.; Shi, H.; Brosseau, J. P.; Wang, H.; Lu, H.; Fang, C.; Zhang, Y.; Liang, L.; Zhou, X.; Wang, C.; Xue, Y.; Cui, Y.; Xu, J., Inhibiting PD-L1 palmitoylation enhances T-cell immune responses against tumours. Nat Biomed Eng 2019, 3 (4), 306-317.

49. Yao, H.; Li, C.; He, F.; Song, T.; Brosseau, J.-P.; Wang, H.; Lu, H.; Fang, C.; Shi, H.; Lan, J.; Fang, J.-Y.; Xu, J., A peptidic inhibitor for PD-1 palmitoylation targets its expression and functions. RSC Chemical Biology 2021. 